\title{
Self similar collapse and the Raychaudhuri equation
}

\author{
Shibendu Gupta Choudhury ${ }^{1, \mathrm{a}}$, Soumya Chakrabarti ${ }^{2, \mathrm{~b}}$, Ananda Dasgupta ${ }^{1, \mathrm{c}}$, Narayan Banerjee ${ }^{1, \mathrm{~d}}$ \\ ${ }^{1}$ Department Of Physical Sciences, IISER Kolkata, Mohanpur, Nadia 741246, India \\ 2 Theory Division, Saha Institute of Nuclear Physics, Kolkata 700064, India
}

Received: 23 October 2019 / Accepted: 11 December 2019 / Published online: 24 December 2019

(c) The Author(s) 2019

\begin{abstract}
The role of the Raychaudhuri equation in studying gravitational collapse is discussed. A self-similar distribution of a scalar field along with an imperfect fluid in a conformally flat spacetime is considered for the purpose. The general focusing condition is found out and verified against the available exact solutions. The connection between the Raychaudhuri equation and the critical phenomena is also explored.
\end{abstract}

\section{Introduction}

It is widely accepted that at the end of its life-cycle, a massive astronomical body undergoes a phase of gravitational collapse. Followed by the pioneering work on an idealized model star collapsing to zero volume by Datt [1] and Oppenheimer and Snyder [2], many attempts have been made to find a more complete and increasingly generalized description of gravitational collapse. For a detailed review of the relevance of gravitational collapse in physics and the open problems, we refer to the references $[3,4]$.

It is not a trivial task to simplify the Einstein field equations enough to guarantee the extraction of an exact solution that can describe the collapsing evolution. This often compels one to resort to the study of simplified systems which can describe the essential physics. Christodoulou studied the global initial value problem for Einstein's equations in the spherically symmetric case with a massless scalar field and showed that the scalar field, depending on the initial collapsing profile, can either converge towards a zero proper volume or bounce, dispersing towards infinity [5-7]. Choptuik studied a similar problem numerically and pointed out the now well known critical phenomena in massless scalar field col-

\footnotetext{
a e-mail: sgc14ip003@iiserkol.ac.in

be-mail: soumya.chakrabarti@saha.ac.in

c e-mail: adg@iiserkol.ac.in

de-mail: narayan@iiserkol.ac.in
}

lapse [8]. Similar critical phenomena were studied by Brady et al. [9] and Gundlach [10]. Throughout the last decade, many attempts have been made to generalize the ideas of critical phenomena for less simplified scenarios. For example, critical phenomena in a collapsing system with angular momentum was studied by Olabarrieta et al. [11]. The critical behavior of a spherically symmetric collision-less matter at the threshold of black hole formation was studied by Olabarrieta and Choptuik [12]. Analytical investigations of the critical collapse problem, and a search for a theoretical explanation for the behavior discovered by Choptuik were carried out by Brady [13], under the assumption that the collapse is self-similar. For a detailed review on the critical phenomena in gravitational collapse we refer to the review by Gundlach and Martin-Garcia [14].

The current work stems from a different motivation - we intend to investigate the role of the Raychaudhuri equation $[15,16]$, in the context of the study of gravitational collapse and critical phenomena. This equation has been used to study relativistic charged collapse by Kouretsis and Tsagas [17]. In the present work we study the evolution of a conformally flat geometry, minimally coupled with a scalar field, along with the presence of a fluid. This specific case of spacetime metric is well-studied for radiating and/or shear-free stars [18-25] and is receiving increasing interest in the context of gravitational collapse quite recently [26-28]. We make the additional assumption that the evolution is self-similar in nature. Gravitational collapse of a self-similar fluid with heat flow in a conformally flat spacetime was studied by Chan et al. [29]. Collapsing models of fluids with the assumption of self-similarity in more general spacetimes were studied in [30-33] and references therein. In this work, we first discuss the usefulness of the Raychaudhuri equation in studying the dynamics of the system. Generic conditions regarding the evolution of the spacetime can be provided using the Raychaudhuri equation. These conditions are useful in the absence of an exact solution. We then work out exact solutions of field equations for some special cases making 
use of some simplifying assumptions. We show the consistency of the conclusions regarding the dynamics from exact solutions, if available, with those arrived at from the Raychaudhuri equation. The role of the Raychaudhuri equation in the context of the critical phenomena is also studied.

The paper is organized as follows. In Sect. 2 we introduce our system and discuss the assumptions involved. The field equations and conservation equations for the system are also presented in this section. The Raychaudhuri equation and focusing condition are briefly discussed in the Sect. 3. In this section, we focus on the role of the Raychaudhuri equation in extracting information about the evolution of spacetime. This section also includes a discussion on the consistency of the results with exact solutions of the field equations, where available, and a connection between focusing condition and the critical phenomena. The final Sect. 4 includes some concluding remarks.

\section{The system}

The spacetime which we consider is a conformally flat spherically symmetric spacetime for which the metric can be written as,

$d s^{2}=\frac{1}{A^{2}(r, t)}\left[-d t^{2}+d r^{2}+r^{2} d \Omega^{2}\right]$,

where $\frac{1}{A^{2}(r, t)}$ is the conformal factor which governs the evolution of the 2-sphere.

The contribution to the energy-momentum tensor comes from a scalar field and a fluid,

$T_{\mu \nu}=T_{\mu \nu}^{\phi}+T_{\mu \nu}^{\text {fluid }}$

where

$T_{\mu \nu}^{\phi}=\partial_{\mu} \phi \partial_{\nu} \phi-g_{\mu \nu}\left[\frac{1}{2} g^{\alpha \beta} \partial_{\alpha} \phi \partial_{\beta} \phi+V(\phi)\right]$,

and

$$
\begin{aligned}
T_{\mu \nu}^{\text {fluid }}= & \left(\rho+p_{t}\right) u_{\mu} u_{\nu}+p_{t} g_{\mu \nu} \\
& +\left(p_{r}-p_{t}\right) \chi_{\mu} \chi_{\nu}+q\left(u_{\mu} \chi_{\nu}+u_{\nu} \chi_{\mu}\right) .
\end{aligned}
$$

$\rho, p_{t}, p_{r}$ and $q$ are the energy density, tangential pressure, radial pressure, and radial heat flux of the fluid respectively; $u^{\mu}=A \delta_{0}^{\mu}$ is the velocity of the fluid and $\chi^{\mu}=A \delta_{1}^{\mu}$ is a unit spacelike vector along the radial direction.

We assume the system to be self-similar of the first kind in nature i.e. the metric admits a homothetic Killing vector [34-36]. In the present case, we write,
$A(r, t)=r B(z), \quad \rho(r, t)=\rho(z), \quad p_{r}(r, t)=p_{r}(z)$,

$p_{t}(r, t)=p_{t}(z), \quad q(r, t)=q(z), \quad \phi(r, t)=\phi(z)$,

where $z=\frac{t}{r}$. With this choice the field equations become self similar (i.e. the only independent variable in these equations is $z$ ).

\subsection{Field equations}

The Einstein field equations (in the units $8 \pi G=1$ ) for the metric (1) with energy momentum tensor (2) can be written as,

$$
\begin{aligned}
& 3 \dot{A}^{2}-3 A^{\prime 2}+2 A A^{\prime \prime}+\frac{4}{r} A A^{\prime} \\
& =\rho+\frac{1}{2} A^{2} \dot{\phi}^{2}+\frac{1}{2} A^{2} \phi^{2}+V(\phi), \\
& 2 \ddot{A} A-3 \dot{A}^{2}+3 A^{\prime 2}-\frac{4}{r} A A^{\prime} \\
& =p_{r}+\frac{1}{2} \phi^{\prime 2} A^{2}+\frac{1}{2} A^{2} \dot{\phi}^{2}-V(\phi), \\
& 2 \ddot{A} A-3 \dot{A}^{2}+3 A^{\prime 2}-\frac{2}{r} A A^{\prime}-2 A A^{\prime \prime} \\
& =p_{t}+\frac{1}{2} A^{2} \dot{\phi}^{2}-\frac{1}{2} \phi^{\prime 2} A^{2}-V(\phi),
\end{aligned}
$$

and

$$
\frac{2 \dot{A}^{\prime}}{A}=\dot{\phi} \phi^{\prime}-\frac{q}{A^{2}}
$$

where dot and prime denote differentiation with respect to $t$ and $r$ respectively.

In terms of the self-similar variable $z$, Eq. (9) can be written as,

$$
\frac{1}{B} \frac{d^{2} B}{d z^{2}}=\frac{1}{2}\left(\frac{d \phi}{d z}\right)^{2}+\frac{q}{2 z B^{2}} .
$$

Equation (10) suggests that if $\phi$ is constant and $q=0, B$ will be proportional to $z$. Similarly, we can write down Eqs. (6)-(8) in terms of $z$ as,

$$
\begin{gathered}
\frac{1}{B} \frac{d^{2} B}{d z^{2}}-\frac{3}{B^{2}}\left(\frac{d B}{d z}\right)^{2}+\frac{2 z}{z^{2}-1} \frac{1}{B} \frac{d B}{d z}+\frac{1}{z^{2}-1} \\
=\frac{\rho}{\left(z^{2}-1\right) B^{2}}-\frac{q\left(1+z^{2}\right)}{2 z\left(z^{2}-1\right) B^{2}}+\frac{V(\phi)}{\left(z^{2}-1\right) B^{2}}, \\
\frac{1}{B} \frac{d^{2} B}{d z^{2}}-\frac{3}{B^{2}}\left(\frac{d B}{d z}\right)^{2}+\frac{2 z}{z^{2}-1} \frac{1}{B} \frac{d B}{d z}+\frac{1}{z^{2}-1} \\
=-\frac{p_{r}}{\left(z^{2}-1\right) B^{2}}+\frac{q\left(1+z^{2}\right)}{2 z\left(z^{2}-1\right) B^{2}}+\frac{V(\phi)}{\left(z^{2}-1\right) B^{2}},
\end{gathered}
$$




$$
\begin{aligned}
& \frac{1}{B} \frac{d^{2} B}{d z^{2}}-\frac{3}{B^{2}}\left(\frac{d B}{d z}\right)^{2}+\frac{4 z}{z^{2}-1} \frac{1}{B} \frac{d B}{d z}-\frac{1}{z^{2}-1} \\
& =-\frac{p_{t}}{\left(z^{2}-1\right) B^{2}}-\frac{q}{2 z B^{2}}+\frac{V(\phi)}{\left(z^{2}-1\right) B^{2}},
\end{aligned}
$$

where Eq. (10) has been used to replace terms containing derivatives of the scalar field. Using Eqs. (11) and (12), one can easily show that,

$\rho=-p_{r}+\frac{q\left(1+z^{2}\right)}{z}$,

while Eqs. (12) and (13) will give,

$\frac{1}{B} \frac{d B}{d z}=\frac{1}{z}+\frac{p_{r}-p_{t}}{2 z B^{2}}-\frac{q}{2 B^{2}}$.

\subsection{Conservation equations}

We shall now write down the conservation equations for this system under the assumption that the energy momentum tensors corresponding to the fluid and the scalar field are conserved independently. The conservation equation for the scalar field yields the wave equation,

$\square \phi-\frac{d V}{d \phi}=0$.

For the present metric (1), this Eq. (16) translates into,

$\ddot{\phi}-\phi^{\prime \prime}-2 \frac{\dot{A}}{A} \dot{\phi}-2 \frac{\phi^{\prime}}{r}+2 \frac{\phi^{\prime} A^{\prime}}{A}+\frac{1}{A^{2}} \frac{d V}{d \phi}=0$.

In terms of the self-similar variable $z$, we have,

$\frac{d^{2} \phi}{d z^{2}}-2 \frac{d \phi}{d z}\left[\frac{1}{B} \frac{d B}{d z}+\frac{z}{1-z^{2}}\right]+\frac{1}{B^{2}\left(1-z^{2}\right)} \frac{d V}{d \phi}=0$.

The conservation equations for the fluid is given by,

$\nabla_{\mu} T_{\text {fluid }}^{\mu \nu}=0$,

from which we will get two non-trivial equations,

$\dot{\rho}+q^{\prime}=\frac{3 \dot{A}}{A}\left(\rho+p_{t}\right)+\frac{\dot{A}}{A}\left(p_{r}-p_{t}\right)+\frac{4 q A^{\prime}}{A}-\frac{2 q}{r}$,

and

$p_{r}^{\prime}+\dot{q}=\left(\frac{3 A^{\prime}}{A}-\frac{2}{r}\right)\left(p_{r}-p_{t}\right)+\frac{A^{\prime}}{A}\left(\rho+p_{t}\right)+\frac{4 q \dot{A}}{A}$.
For the self similar case, if we use Eq. (14), both Eqs. (20) and (21) yield the same equation as,

$\frac{d \rho}{d z}-z \frac{d q}{d z}=\frac{2}{B} \frac{d B}{d z}\left(\rho+p_{t}\right)+\frac{\left(1-3 z^{2}\right) q}{z B} \frac{d B}{d z}+2 q$.

\section{Raychaudhuri equation}

For a timelike congruence having velocity vector $u^{\mu}$, the Raychaudhuri equation is given by $[15,16]$,

$\frac{d \theta}{d \tau}=-\frac{1}{3} \theta^{2}+\nabla_{\mu} a^{\mu}-\sigma_{\mu \nu} \sigma^{\mu \nu}+\omega_{\mu \nu} \omega^{\mu \nu}-R_{\mu \nu} u^{\mu} u^{\nu}$,

where $\theta=\nabla_{\mu} u^{\mu}$ is the expansion scalar, $\tau$ is affine parameter, $\sigma_{\mu \nu}=\nabla_{(\nu} u_{\mu)}-\frac{1}{3} h_{\mu \nu} \theta+a_{(\nu} u_{\mu)}$ is the shear tensor where $h_{\mu \nu}$ is the spatial metric, $\omega_{\mu \nu}=\nabla_{[\nu} u_{\mu]}-a_{[\nu} u_{\mu]}$ is the rotation tensor, $a^{\mu}=u^{\nu} \nabla_{\nu} u^{\mu}$ is the acceleration vector and $R_{\mu \nu}$ is the Ricci tensor.

\subsection{Focusing condition}

We have chosen a comoving observer, so that $u^{\alpha}=A \delta_{0}^{\alpha}$. As the metric is conformally flat, the shear term and rotation term will vanish.

Now, we know that the congruence will focus [37] within a finite affine parameter value if,

$\frac{d \theta}{d \tau}+\frac{1}{3} \theta^{2} \leq 0$,

which, with Eq. (23), leads to,

$R_{\mu \nu} u^{\mu} u^{\nu} \geq \nabla_{\mu} a^{\mu}$.

Here the left hand side of the expression is related to the matter sector via the Einstein equations and the right hand side is the divergence of acceleration. Focusing may be forbidden when the divergence of acceleration opposes the gravitational attraction and its contribution dominates over that due to the matter part. If the divergence of acceleration term is sufficiently high, the evolution may lead to a complete dispersal.

For the metric (1), the condition (25) in terms of the conformal factor $A(r, t)$ is given by,

$\frac{\partial}{\partial t}\left(\frac{\dot{A}}{A}\right) \geq 0$.

For the self similar case, the above condition (26) becomes,

$\frac{d}{d z}\left(\frac{1}{B} \frac{d B}{d z}\right) \geq 0$. 
3.2 Raychaudhuri equation and the dynamics of spacetime

It is difficult to find exact solutions of the field equations for our system without any simplifying assumptions. The Raychaudhuri equation can be applied to provide generic conditions, regarding the dynamics of the spacetime. These conditions may lead us to some useful information about the evolution. We will now try to find such conditions.

We have the condition for focusing (27) from the Raychaudhuri equation as,

$\frac{d}{d z}\left(\frac{1}{B} \frac{d B}{d z}\right) \geq 0$

Using Eq. (15) this condition can be written as,

$\frac{d}{d z}\left(\frac{p_{r}-p_{t}}{2 z B^{2}}-\frac{q}{2 B^{2}}\right) \geq \frac{1}{z^{2}}$

These generic conditions like (28) or (29), may appear useful. These are generic conditions if satisfied by the conformal factor or the energy momentum tensor components, will lead to the formation of a singularity. Thus, there is an important role of these conditions in dictating the dynamics of the spacetime. Let us illustrate this point with a simple but very important example. If the fluid is a perfect isotropic fluid having no radial heat flux, the left hand side of the condition (29) is equal to zero. Thus, the condition is satisfied only when $z \rightarrow \infty$ which means that there will be a central singularity at $r=0$ or a singularity forms as $t \rightarrow \infty$ which is inconsequential.

Thus, the possibility of the formation of a singularity at a finite future or the avoidance of a central singularity will depend on pressure anisotropy or heat flux.

We will discuss a few more examples. The condition (28) or (29) can be recast into different forms using the field equations. For example, if we use Eqs. (10) and (15), the condition (28) can be written as,

$\frac{1}{2}\left(\frac{d \phi}{d z}\right)^{2}+\frac{q}{2 z B^{2}} \geq\left(\frac{1}{z}+\frac{p_{r}-p_{t}}{2 z B^{2}}-\frac{q}{2 B^{2}}\right)^{2}$.

Now, let us consider the case where the scalar field is absent or a constant (equivalent to a cosmological constant) and $q=0$. In this case the above condition (30) will be satisfied only when

$\frac{1}{z}+\frac{p_{r}-p_{t}}{2 z B^{2}}=0$

which implies $\frac{d B}{d z}=0$ [using Eq. (15)] and there will not be any evolution of the spacetime. Therefore, we can conclude that formation of a singularity in this case can be avoided.
However, $q \neq 0$ or $\frac{d \phi}{d z} \neq 0$ or both may lead to the formation of a singularity. If we have a large rate of change of the scalar field, compared to the other terms, present in the condition (30), singularity formation is inevitable.

Let us discuss another example where $\frac{d \phi}{d z}=0$ and $p_{r}=$ $p_{t}$. The condition (30) then translates into,

$\frac{q^{2}}{4 B^{4}}-\frac{3 q}{2 z B^{2}}+\frac{1}{z^{2}} \leq 0$

which gives,

$$
\frac{(3-\sqrt{5}) B^{2}}{z} \leq q \leq \frac{(3+\sqrt{5}) B^{2}}{z} .
$$

Thus, there will be singularity formation only when the heat flux of the fluid satisfies this constraint (33).

\subsection{Exact solutions and the Raychaudhuri equation}

We have not used exact solutions of the field equations in our discussion so far. It is worthwhile to check the consistency of the conclusions arrived at using the Raychaudhuri equation with those using exact solutions whenever available. For many special cases, i.e., with various sources, exact solutions for the metric can be found out. Some of them are listed in the Appendix A. With an exact solution, one can explicitly find whether there is a collapse or an expansion and whether the collapse, if there is any, results in a singularity. We have checked that in all such cases the conclusions are consistent with those obtained from the focusing condition (27) found out from the Raychaudhuri equation. One such nontrivial example is described in detail below.

We will discuss the case where a scalar field and a fluid both are present and we make an assumption that $p_{r} \neq p_{t}$ but $q=0$. For details, see Appendix A.5. The solution for $B$ and $A$ in this case are respectively given by,

$B=\left(F z^{2 \beta}-k\right)^{\frac{1}{2 \beta}}$

and

$A=\left(F t^{2 \beta}-k r^{2 \beta}\right)^{\frac{1}{2 \beta}}$

where $\beta$ is a constant given by $p_{t}=\beta p_{r}$. For $\beta>0$, there may be a zero proper volume singularity when $t \rightarrow \infty$ and/or $r \rightarrow \infty$, which can be excluded from the discussion. There will be a dispersal in this case at $z=\left(\frac{k}{F}\right)^{\frac{1}{2 \beta}}$. When $\beta<0$, formation of a singularity at $z=\left(\frac{k}{F}\right)^{\frac{1}{2 \beta}}$ is inevitable. In this 
case, a dispersal occurs at $t=0$. There may be a dispersal at $r=0$ which is not physically significant.

Using the solution for $B$ [Eq.(34)], one can show,

$$
\frac{d}{d z}\left(\frac{1}{B} \frac{d B}{d z}\right)=-\frac{2 F^{2} \beta z^{(4 \beta-2)}}{\left(F z^{2 \beta}-k\right)^{2}}+\frac{F(2 \beta-1) z^{(2 \beta-2)}}{\left(F z^{2 \beta}-k\right)}
$$

When $z \rightarrow\left(\frac{k}{F}\right)^{\frac{1}{2 \beta}}$, the first term in the right hand side of the above expression dominates. Only when $\beta<0$, the condition (28), obtained using the Raychaudhuri equation, is fulfilled in this region and a singularity within finite $z$ is unavoidable. On the other hand if $\beta>0$, a dispersal will occur in this region. Singularity formation as $z \rightarrow \infty$ is a possibility in this case. For $\beta<0$, it is easy to show that the right hand side of the Eq. (36) must be negative as $z \rightarrow 0$ which makes a dispersal possible here.

\subsection{Critical phenomena and the Raychaudhuri equation}

From the exact solutions in Appendix A, we have found that collapse or dispersal correspond to the quantity $\frac{d}{d z}\left(\frac{1}{B} \frac{d B}{d z}\right)$ being positive or negative, respectively. Therefore, a transition from collapse to dispersal or vice versa will be accompanied with a change of the sign of this quantity. Thus, a relation between focusing condition and the critical phenomena seems to be indicated. We will illustrate this point using the example discussed in the previous section.

In this example, let us take the $z \rightarrow\left(\frac{k}{F}\right)^{\frac{1}{2 \beta}}$ into consideration. When $\beta$ is negative a singularity forms at $z=\left(\frac{k}{F}\right)^{\frac{1}{2 \beta}}$ while dispersal occurs when $\beta$ is positive. Thus, $\beta$ can be treated as a critical parameter. We have seen in the previous section that positive or negative $\beta$ corresponds to the situation when the quantity $\frac{d}{d z}\left(\frac{1}{B} \frac{d B}{d z}\right)$ is negative or positive respectively as $z \rightarrow\left(\frac{k}{F}\right)^{\frac{1}{2 \beta}}$. This shows that the critical parameter $\beta$ determines the signature of $\frac{d}{d z}\left(\frac{1}{B} \frac{d B}{d z}\right)$ which in turn determines the focusing (collapse) or dispersal of the spherically symmetric distribution and the critical value of $\beta$ is zero.

\section{Conclusion}

We have found the focusing condition (28) for a self similar matter distribution in a conformally flat spherically symmetric spacetime. Although the spacetime, we have considered, has a stringent symmetry requirement, the matter distribution is quite general and includes anisotropic fluid pressure, heat flux, as well as a minimally coupled scalar field. The nature of the conclusions drawn from this condition is verified against quite a few exact solutions that are available, and one of them is discussed in detail.
Thanks to the investigations as in references [8-10] and others, it is now well known that a scalar field collapse may have a critical phenomenon associated with it. The condition developed from the Raychaudhuri equation helps visualizing the critical phenomena in general. This remains valid even when the matter distribution includes a fluid. We have discussed one example, where exact solution is available, in detail. In this example, we could see the existence of a critical parameter, and could also determine the critical value $(\beta=0)$. It is intriguing to note that the parameter has nothing to do with the scalar field in this case and is determined only by the fluid, as $\beta$ is simply a parameter connecting the radial and transverse fluid pressure. With the help of this example we have explicitly discussed the relation between the focusing condition and the critical phenomena.

Acknowledgements Shibendu Gupta Choudhury (SGC) thanks Council of Scientific and Industrial Research, India for the financial support. SGC would like to thank Prof. Sayan Kar, Indian Institute of Technology, Kharagpur for guidance and useful discussions.

Data Availability Statement This manuscript has no associated data or the data will not be deposited. [Authors' comment: The authors neither used nor generated any data while performing this work.]

Open Access This article is licensed under a Creative Commons Attribution 4.0 International License, which permits use, sharing, adaptation, distribution and reproduction in any medium or format, as long as you give appropriate credit to the original author(s) and the source, provide a link to the Creative Commons licence, and indicate if changes were made. The images or other third party material in this article are included in the article's Creative Commons licence, unless indicated otherwise in a credit line to the material. If material is not included in the article's Creative Commons licence and your intended use is not permitted by statutory regulation or exceeds the permitted use, you will need to obtain permission directly from the copyright holder. To view a copy of this licence, visit http://creativecomm ons.org/licenses/by/4.0/.

Funded by $\mathrm{SCOAP}^{3}$.

\section{Appendix A: A few exact solutions}

Some examples of exact solutions with the assumption of self similarity, for a conformally flat, spherically symmetric spacetime in presence of a scalar field and a fluid, imperfect in general, are given here. Some of the solutions included are already there in the literature.

\section{Appendix A.1: Massless scalar field}

Let us first consider the simplest case where the contribution to the matter part comes only from a massless scalar field. From the Eq. (15) we have,

$$
\frac{1}{B} \frac{d B}{d z}=\frac{1}{z}
$$


which gives,

$B=m z$,

where $m$ is constant of integration. Thus, the solution for $A(r, t)$ is,

$A=r B=m t$.

But we should note that with Eq. (A.1), Eqs. (11), (12) and (13) all yield,

$\frac{3}{z^{2}\left(z^{2}-1\right)}=0$

which is inconsistent for finite $z$ values. Thus, a consistent solution of the field equations with a massless scalar field as matter source is not possible under the mentioned assumptions.

Appendix A.2: Scalar field with a non-zero potential

Even if we include a potential in the energy momentum tensor of the scalar field, we have the same solutions for $B$ and $A$ as in the previous case [Eqs. (A.2) and (A.3) respectively]. Here with Eq. (A.2), Eqs. (11), (12) and (13) all yield,

$V(\phi)=3 m^{2}$

Therefore, the potential must remain constant.

From Eq. (10) we have,

$\phi=$ constant

which is consistent with the Eq. (18). Thus, the net energy momentum tensor effectively behaves like that of a cosmological constant.

From Eq. (A.3), we can conclude that there is a singularity only when $t$ approaches infinity where the scale factor (inverse of $A$ ) becomes zero which signifies an ever collapsing solution. At $t=0, A=0$ i.e. the scale factor becomes infinite which signifies a dispersal.

Appendix A.3: Scalar field along with a perfect fluid

If we include perfect isotropic fluid ( $p_{r}=p_{t}$ and $q=0$ ) along with the scalar field, the solution for $B$ will not change as dictated by Eq. (15). From Eq. (14) we have,

$\rho=-p$

$\rho, p, \phi$ and $V(\phi)$ will remain constant in this case. Thus the matter turns out to be the cosmological constant for a con- sistent solution. Clearly, the conclusions in this case remain the same as in the previous case.

In the examples, discussed so far we have not observed any possibilities of singularity formation within finite time and the matter turns out to be the cosmological constant.

Appendix A.4: Fluid with isotropic pressure and radial heat flux

This particular case, with $p_{r}=p_{t}$ and $q \neq 0$, has already been studied by Chan et al. [29]. They found that there is a singularity formation due to collapse as $t \rightarrow 0$. In this case from Eqs. (15) and (10) we have,

$\frac{z}{B} \frac{d^{2} B}{d z^{2}}+\frac{1}{B} \frac{d B}{d z}-\frac{1}{z}=0$.

The solution for $B$ is given by,

$B=\frac{C z^{2}+2 D}{2 z}$,

where $C$ and $D$ are constants of integration. From the above Eq. (A.9) we have,

$A=\frac{C t^{2}+2 D r^{2}}{2 t}$

The expression for $A$ confirms that there is a zero proper volume singularity at $t=0$. The scale factor also becomes zero when $t \rightarrow \infty$ and/or $r \rightarrow \infty$. These possibilities are not worth considering. There may be a dispersal at $z^{2}=\frac{t^{2}}{r^{2}}=$ $-\frac{2 D}{C}$ only if $C$ and $D$ are of opposite signs.

Appendix A.5: Scalar field along with an anisotropic fluid

In this case, we have $p_{r} \neq p_{t}$ but $q=0$. Now, from Eqs. (14) and (15) we have,

$\rho=-p_{r}$

and

$\frac{1}{B} \frac{d B}{d z}=\frac{1}{z}+\frac{p_{r}-p_{t}}{2 z B^{2}}$.

The same equation of state as in Eq. (A.11) was obtained by Brandt et al. [32] from the requirement of self-similarity even with a relaxation of the conformally flat condition. In this case, the conservation equation for the fluid [Eq. (22)] takes the form,

$\frac{d p_{r}}{d z}=\frac{2}{B} \frac{d B}{d z}\left(p_{r}-p_{t}\right)$. 
It is difficult to find a solution for the conformal factor in this case without further simplifications. Thus, we will assume that the tangential pressure is proportional to radial pressure i.e. $p_{t}=\beta p_{r}$. Brandt et al. worked with a similar assumption for a spherically symmetric spacetime which is not conformally flat [31]. With this assumption, solving Eq. (A.13) we have,

$p_{r}=n B^{2(1-\beta)}$,

where $n$ is constant of integration. If we replace this in Eq. (A.12), the solution for $B$ comes out as,

$B=\left(F z^{2 \beta}-k\right)^{\frac{1}{2 \beta}}$,

where $F$ is constant of integration and $k=\frac{n(1-\beta)}{2}$. Consequently, the solution for $A$ is,

$A=\left(F t^{2 \beta}-k r^{2 \beta}\right)^{\frac{1}{2 \beta}}$

From Eq. (A.11) one can see that the radial pressure of the fluid comes out to be negative. For consistency, we may consider the fluid to be a viscous fluid.

\section{References}

1. B. Datt, Z. Phys. 108, 314 (1938)

2. J.R. Oppenheimer, H. Snyder, Phys. Rev. 56, 455 (1939)

3. P.S. Joshi, Global aspects in Gravitation and Cosmology, International Series of Monographs on Physics (Clarendon Press (OUP), Oxford, 1993)

4. P.S. Joshi, Pramana 55, 529 (2000)

5. D. Christodoulou, Commun. Math. Phys. 109, 591 (1987)

6. D. Christodoulou, Commun. Math. Phys. 109, 613 (1987)

7. D. Christodoulou, Ann. Math. 140, 607 (1994)

8. M.W. Choptuik, Phys. Rev. Lett. 70, 9 (1993)

9. P.R. Brady, C.M. Chambers, S.M.C.V. Goncalves, Phys. Rev. D 56, R6057 (1997)
10. C. Gundlach, Phys. Rev. Lett. 75, 3214 (1995)

11. I. Olabarrieta, J.F. Ventrella, M.W. Choptuik, W.G. Unruh, Phys. Rev. D 76, 124014 (2007)

12. I. Olabarrieta, M.W. Choptuik, Phys. Rev. D 65, 024007 (2001)

13. P.R. Brady, Class. Quantum Gravity 11, 1255 (1995)

14. C. Gundlach, J.M. Martin-Garcia, Living Rev. Relat. 10, 5 (2007)

15. A.K. Raychaudhuri, Phys. Rev. 98, 1123 (1955). Reprinted as a 'Golden Oldie', Gen. Relativ. Gravit. 32, 749 (2000)

16. J. Ehlers, Akad. Wiss. Lit. Mainz, Abhandl. Math.-Nat. K1. 11, 793 (1961). Translation: J. Ehlers, Gen. Relativ. Gravit. 25, 1225 (1993)

17. A.P. Kouretsis, C.G. Tsagas, Phys. Rev. D 82, 124053 (2010)

18. M.M. Som, N.O. Santos, Phys. Lett. A 87, 89 (1981)

19. S.R. Maiti, Phys. Rev. D 25, 2518 (1982)

20. B. Modak, J. Astrophys. Astron. 5, 317 (1984)

21. A. Banerjee, S.B.D. Choudhury, B.K. Bhui, Phy. Rev. D 40, 670 (1989)

22. L.K. Patel, R. Tikekar, Math. Today IX, 19 (1991)

23. D. Schafer, H.F. Goenner, Gen. Relativ. Gravit. 42, 2119 (2000)

24. B.V. Ivanov, Gen. Relativ. Gravit. 44, 1835 (2012)

25. L. Herrera, G. Le Denmat, N.O. Santos, A. Wang, Int. J. Mod. Phys. D 13, 583 (2004)

26. A.I.M. Hamid, R. Goswami, S.D. Maharaj, Class. Quantum Gravity 31, 135010 (2014)

27. S. Chakrabarti, N. Banerjee, Eur. Phys. J. C 77, 166 (2017)

28. N. Banerjee, S. Chakrabarti, Phys. Rev. D 95, 024015 (2017)

29. R. Chan, M.F.A. da Silva, J.F.V. da Rocha, Int. J. Mod. Phys. D 12, 347 (2003)

30. C.F.C. Brandt, L.M. Lin, J.F.V. da Rocha, A.Z. Wang, Int. J. Mod. Phys. D 11, 155 (2002)

31. C.F.C. Brandt, M.F.A. da Silva, J.F.V. da Rocha, R. Chan, Int. J. Mod. Phys. D 12, 1315 (2003)

32. C.F.C. Brandt, R. Chan, M.F.A. da Silva, J.F.V. da Rocha, Int. J. Mod. Phys. D 15, 1407 (2006)

33. R. Chan, M.F.A. da Silva, C.F.C. Brandt, Int. J. Mod. Phys. D 23, 1450056 (2014)

34. R. Maartens, S.D. Maharaj, Class. Quantum Gravity 3, 1005 (1986)

35. S. Moopanar, S.D. Maharaj, J. Eng. Math. 82, 125 (2013)

36. S. Moopanar, S.D. Maharaj, Int. J. Theor. Phys. 49, 1878 (2010)

37. E. Poisson, A Relativist's Toolkit: The Mathematics of Black-Hole Mechanics (Cambridge University Press, Cambridge, 2004) 